\title{
La Russe en deuil
}

D. Herrenschwand

Natürlich musste es heute regnen, der Schnee fiel armselig in sich zusammen und sie würde in dem braunweissen Matsch zum Bahnhof laufen, um ihre Schwiegermutter abzuholen.

Dabei hatte sie sich vorgestellt, wie sie die Alte durch prunkvoll saubere Strassen führe, ihr den blauen Himmel über der weiss beladenen Altstadt zeige.

So etwas gab es nicht in Marseille!

Gestern noch hatte sie in der tief verschneiten Stadt das Gefühl gehabt, überall die Schneekönigin zu sehen, und noch abends spät war sie über die Brücke in die weihnachtlich geschmückten Gassen gelaufen.

Sie hatte das Lied von der stillen Nacht summen können, ohne dass ihr wie sonst Tränen der Rührung - oder war es Verlorenheit? - über die Wangen gelaufen wären. Dieses Lied hatte ihr schon als kleines Mädchen feuchte Augen beschert.

Lang war das her - sie straffte ihren Rücken.

Der Schnee hatte sie ruhig gemacht, sie hatte sogar gelassen zusehen können, wie Pierre den viel zu grossen Christbaum in der Küche mit brutalen Axthieben stumm zurechtstutzte und sich dabei ein Glas Cognac nach dem andern einschenkte.

Sie hätte die Flasche eigentlich für den Heiligen Abend aufheben wollen, hatte sie günstig erstanden. Aber das Schweigen zwischen ihnen war besser zu ertragen als die Streitereien.

Pierre hatte nur knapp gefragt, ob für die Ankunft seiner Mutter alles vorbereitet sei und Noelle an die zusätzliche Bettdecke gedacht habe. Seufzend zwängte sich Noelle in ihre Stiefel und warf einen kurzen Blick ins Schlafzimmer, das sie für die Schwiegermutter geräumt hatte - Pierre und sie würden in den nächsten zwei Wochen in der Kammer neben der Küche schlafen.

Er auf einer Matratze am Boden, sie auf der alten Couch, die sonst in der Veranda stand.

Sie würde frieren, das Öfchen war zu schwach, um ein Zimmer wohlig zu erwärmen, aber die Tagesdecke mit den Brüsseler Spitzen lag blütenweiss auf dem grossen Doppelbett für die Alte ausgebreitet.

Unschuldslager, murmelte sie bitter und sah die zerwühlten Laken ihrer durchspielten Nächte mit Pierre, sah sich zufrieden schnurrend in den Kissen liegen und darauf warten, dass er ihr den Kaffee bringe, sie küsse.
Wie lange war das her?

Damals jedenfalls war er verliebt gewesen in ihre langen Haare, hatte sorgsam Nadel um Nadel aus dem geflochtenen Kranz gezogen, bis ihre Locken wie ein fliessender Vorhang über Gesicht, Nacken und Rücken geglitten waren und er sein Gesicht darin vergraben konnte, weil er nicht genug bekam von dem Duft, von ihrem Geruch.

Damals hatte sie es nicht nötig gehabt, schamvoll einen dicken Morgenrock um ihren Körper zu schlingen, damit keiner sähe, wie sterbensweiss und schlaff er sei. Doch jetzt konnten alle Öfen der Welt die Kälte in ihrem Bett nicht vertreiben. Starr lagen sie da, bemüht, jeder auf seiner Kante zu bleiben.

Noelle lag begraben unter dem Schutt ihres Alltags und nie würde sie sein Zusammenzucken vergessen, wenn ihr klammer Fuss wie zufällig seine Wade streifte.

Sie zog die Wohnungstüre heftig hinter sich $\mathrm{zu}$.

Leise rieselte Kalk aus dem Verputz der Türfüllung, sie achtete nicht darauf und sah sich vor, dass sie auf den schadhaften Steinstufen der Treppe nicht stürzte.

Die Äste der verschneiten Birken hatten gestern gnädig den Blick auf den schäbigen Charme des hohen Hauses am Fluss verschleiert, in dem sie wohnten, weil die Mieten billig waren.

Aber heute, im trüben Zwielicht, sah es besonders baufällig aus. Die nackten Zweige hatten ihren Stolz verloren, peitschten unter Windstössen erbarmungslos die blassgelben Mauern.

Brüchig wirkte das Haus geradezu, die Alte würde es zweifellos bemerken und eine ihrer bissigen Bemerkungen fallenlassen, Noelle wusste es.

Zum Glück hatte sie den Hut aufgesetzt, eine schwarze Pelzkappe, die sie letzte Woche auf dem Weihnachtsmarkt gekauft hatte.

Noelle fand die enganliegende Mütze sehr kleidsam, auch wenn das Fell schon ein bisschen abgeschabt war. Der Mann am Trödelstand hatte ihr sogar einen Spiegel hingehalten, damit sie sehen konnte, wie gut sie ihr stand.

Sie kannten den Kollegen seit langem, Pierre und er hatten schon manches Jahr ihre Stände nebeneinander, sich die kalten Füsse vertreten und einen Glühwein geholt, wenn sie ganz durchfroren waren. 
Sie lebten vom Verkauf alter Möbel und Bilder, die sie an Märkten und Messen anboten, nachdem Pierre sie restauriert hatte.

Das konnte er, und sie staunte immer wieder, mit welcher Zärtlichkeit er, der ehemalige Hafenarbeiter, die kostbaren Kirschbaumtische behandelte, die alten Sessel sorgfältig mit neuem Brokat überzog.

Er kannte sich aus, war ein Fachmann geworden in den Jahren, und sie hatte ihm geholfen, hatte altes Silber geputzt und Porzellangeschirr gewaschen, das sie irgendwo erstanden hatte, um es weiterzuverkaufen.

Doch das Geschäft lief nicht mehr - auch andern Händlern ging es so. Man redete über die Krise in den langen Stunden, es kam ja keiner und man hatte Zeit.

Pierre aber sprach nicht.

Verbissen sass er in der Werkstatt, wenn kein Markt war, auch keine Auktion.

Ruhelos ging er hin und her, und einmal hatte er einen Hammer durch den Raum geschleudert. Einfach so.

Das war nicht mehr der schöne, dunkle Mann, den sie vor zwanzig Jahren auf einer Reise nach Südfrankreich im Zug getroffen hatte, der sie mit seiner verführerischen Sprache, dem unwiderstehlichen Lächeln und dem Spiel seiner grossen Hände so fasziniert hatte, dass ihr in Marseille, wo der Zug stillstand, alles ganz klar war.

Sie würden zusammenbleiben.

Nicht einmal die kleinen Hinterzimmer der Hafenkneipe, die er bewohnte, hatten sie erschreckt.

Nicht das kahle Mobiliar und nicht die Geräusche der Strasse, das kreischende Lachen der Dirnen, das Gebrüll der Betrunkenen. Nie zuvor hatte sie Köstlicheres geschmeckt als die Fischsuppe, die sie spätabends in der dämmerigen Kneipe assen, und sie hatte sich lachend zeigen lassen, wie man die scharfe Knoblauchpaste erst auf ein Stück des weissen Brotes schmiert, bevor man es in die Suppe tunkt und dann rasch zum Mund führt.

Seine Mutter trafen sie erst nach ein paar Tagen.

Königlich grollend sass sie auf abgeschabtem Sessel in ihrer grossen Küche, hielt Hof über die Schar ihrer Kinder.

Pierre, der einzige Sohn, küsste ihre Hände mit den billigen Goldringen, während sie Noelle durchdringend musterte.

«Diese also - nun ja, wenn es sein muss ... » hatte sie gesagt.

Noelle lachte sich den Kloss aus dem Hals, sie war jung, die ganze Welt gehörte ihr und Pierre!
Sie heirateten und lebten in der Stadt, aus der sie kam, weil er sich nichts brennender wünschte, als seiner Herkunft den Rücken zu kehren.

Die Wochen im Sommer und zu Weihnachten, wenn die Schwiegermutter sie besuchte, versuchte Noelle zu überstehen und ganz rasch zu vergessen, auch die verwirrte Kränkung darüber, dass ihr Mann sie in dieser Zeit nie berührte.

Wenn sie dann gegangen war, wurde alles wie vorher.

Pierre, unersättlicher Liebhaber, bedachte Noelle mit all den liebevollen Kleinigkeiten, die ihr das Gefühl gaben, begehrt zu sein.

Doch die Zeit floss dahin, stetig wie das Wasser des Flusses, an dem sie wohnten.

Als sie mit den Jahren kinderlos blieben, wuchs die Verachtung der Alten ins Grenzenlose.

Wann merkte Pierre, dass er kein reicher Mann werden würde?

Wann schlich die Angst in seinem Leib hoch - vor der Armut, die ihn einholen würde, vor dem Dreck und der Hitze im Hafen?

Seine ohnmächtigen Zornesausbrüche machten ihr Angst, sie dachte ja schon lange daran, ihn zu verlassen.

Aber sie konnte es nicht, ohne Geld und Beruf!

Noelle hatte den Bahnhof zu früh erreicht, stand jetzt da und wartete auf die Ankunft des Zuges.

Als er mit kreischenden Bremsen stillstand, öffnete die Schwiegermutter als erste die Tür und stieg, mit Taschen und Einkaufstüten beladen, einen Wollschal um das Vogelgesicht geschlungen, schwerfällig aus. Dann blickte sie umher und sah Noelle.

«Mon dieu ... tu as vraiment l'air d'une Russe en deuil ...», war das einzige, was sie schweratmend hervorbrachte. Sie verlieh dem Satz eine absichtlich verletzende Bedeutung, indem sie ihre Stimme tief absinken liess.

Noelle wollte ihre Verwirrung um keinen Preis zeigen, nahm schweigend den Arm der Alten und führte sie aus dem Gewimmel auf dem Bahnsteig in die Unterführung. Sie passte ihren Schritt dem Humpeln der alten Frau an und warf verstohlene Blicke in die Schaufensterscheiben.

Trauernde Russin - sie sah sich, eine stattliche, nicht mehr junge Frau, deren tief in die Stirn gezogene Fellkappe das traurige Gesicht nicht verbarg.

Der wadenlange Mantel, die Taille eng vom breiten Gürtel umschlungen, Lederstiefel, ... die Alte mochte sie wirklich so sehen.

Noelle trauerte ja, doch das würde sie nicht verstehen, brauchte es auch nicht zu wissen. 
Eine Russin war sie nicht. Oder etwa doch?

Während sie im Tram sass, das Geschwätz von Pierres Mutter sich über ihr ergoss, ohne dass sie dessen Inhalt aufnahm, liess Noelle ihre Gedanken weiterschweifen, spürte dem fremden Klang des Wortes «Tundra» nach, das sich nicht aus ihrem Kopf verbannen liess und wusste plötzlich sehr genau, was sie tun musste. «Wir treffen Pierre im Café, er hatte noch ein Geschäft zu machen», sagte sie leichthin, die Alte war zufrieden.

Pierre sass an einem der kleinen Tische, ein Glas Wein vor sich, und erwartete aufgeregt seine Mutter, die sich ihm um den Hals warf. «Hier ist deine Mutter, Pierre, ich gehe ...» sagte Noelle nicht unfreundlich und verliess erhobenen Kopfes das Lokal. Sie eilte nach Hause, suchte nach dem kleinen Schlüssel zum verborgenen Fach des Sekretärs, fand ihn endlich versteckt in der Zuckerbüchse.
Sie öffnete rasch das Geheimfach und fand unter Papieren und losen Fotografien, woran sie sich im Tram erinnert hatte:

Das Erbstück ihres Grossvaters, die russische Tabatière aus beschlagenem Silber.

Sie blies den Staub weg, hauchte und rieb, bis das Kästchen wieder glänzte. Dann packte sie es sorgsam mit einigen Kleidern in ihre Reisetasche.

Warum hatte sie das Stück Pierre nie gezeigt? Weil er es sogleich verkauft hätte?

Sie wusste, dass die alte Dose kostbar war, doch jetzt, in diesem Augenblick, schien sie ihr unschätzbar, denn sie würde ihr einen neuen Anfang ermöglichen - wie auch immer!

Sie liess ihren Schlüsselbund auf dem Küchentisch zurück und zog entschlossen die Türe hinter sich ins Schloss.

\section{Fristenregelung, Stammzellen- und Gen- forschung, Euthanasie usw., eine Herausforde- rung an jeden und die Weltgemeinschaft}

\section{Ein Versuch}

\section{J. Dossenbach}

Korrespondenz:

Dr. med. Josef Dossenbach Bahnhofstrasse 18 CH-6300 Zug
Am 2. Juni haben wir über die Fristenregelung abgestimmt; der Bundesrat will im Juni einen Gesetzesentwurf über die Forschung an Stammzellen veröffentlichen; über die Genforschung und die Sterbehilfe berichten die Medien fast wöchentlich; die pränatale Diagnostik harrt einer gesetzlichen Regelung und an die Retortenbabies haben wir uns schon gewöhnt.

Wir Menschen wurden fähig, in einer Weise in die Natur einzugreifen, die uns vor schwere ethische Konflikte stellt. Wie die meisten meiner Kollegen hat mich diese Problematik mein ganzes Leben begleitet: Als Dissertant, der in seiner Untersuchung «über die Praxis der Begutachtung der Schwangerschaftsfähigkeit in der Psychiatrischen Poliklinik Zürich von 1933 bis 1978» zum Schluss kam, dass diese Praxis durch den Zeitgeist bestimmt ist und nicht durch die psychiatrische Diagnostik; als Psychiater, der Schwangerschaftsfähigkeitsgutachten verfasste; als Vater einer Tochter, die in Erbbiologie forscht; als naher Verwandter einer Familie, die sich gegen den Abbruch einer Schwangerschaft wehrte und noch heute die finanziellen Folgen bewundernswert trägt; als Christ, der einerseits die unbeirrbare Konsequenz des Papstes in seinem Denken und Handeln bewundert, aber gleichzeitig ein liberaler Revoluzzer geblieben ist; als Arzt, der viele Krebskranke und einige Multiple-Sklerose-Patienten in ihren letzten Wochen und Tagen begleitete. Vier von ihnen verkürzten ihr Leiden mit den Mitteln, die sie von «Exit» bezogen. Ich war ihnen nahe und verstand sie, obwohl ich mir für mich vornahm, 
mein Leben nie selber zu beenden. Ich schildere dies so persönlich, weil wir alle in diesen Konflikten leben und ihre Lösung nicht den Politikern und Ethikern überantworten können. Sie betreffen unser Leben und das unserer Kinder!

Was ist Leben? Der Moment, in dem es auf dieser Erde entstand, bleibt ebenso ein Rätsel, wie die Frage, wie sich solche hochkomplexen Strukturen entgegen dem Gesetz der Entropie aufbauen und erhalten konnten. Wir erkennen aber zwei entgegengesetzte Prinzipien, die in der Natur walten: Das eine formt geschlossene Systeme von grossartiger Vielfalt und das andere strebt nach dem Kräfteausgleich, nach dem Zerfall dieser Systeme in die Strukturlosigkeit und Undifferenziertheit. Wir erkennen auch, dass Strukturen nur mit grossen Energien aufrechterhalten werden können. Die leblose Materie, indem sie Energien bindet, die lebendige, indem sie sich ständig Energien zuführt. Zum ständigen Aufbau und Erhalt und zu deren Lebendigkeit beziehen die Pflanzen ihre Stoffe aus der unbelebten Materie, dem Boden und die Energie von der Sonne, die Tiere und wir Menschen beziehen Baustoffe und Energie aus dem Einverleiben anderer lebendiger Organismen, aus Pflanzen und Tieren. Allem strukturiertem Sein ist das Gesetz von Werden und Vergehen eigen, dem die Natur durch ein weiteres grossartiges Rätsel zu entrinnen sucht, nämlich im Bestreben, sich und seine Art zu erhalten, sich fortzupflanzen, und zwar nicht kreuz und quer, Rose und Margarite, sondern jedes in seiner Art. Warum eigentlich?

Allen diesen Gesetzen der Natur ist der Mensch «unterworfen» oder darf sie bewusst geniessen. Der Verstand, der sich ihm selber bewusst macht, hat aber auch die Fähigkeit, in die Natur einzugreifen: Er kann Leben fördern; er kann Leben z.B. durch Kreuzen verändern; er kann Leben z.B. mit der Medizin erhalten; er kann Leben verhindern und er kann Leben vernichten. Diese Fähigkeit beinhaltet eine grosse Verantwortung. Um ihr gerecht zu werden, haben wir zwei Instrumente: Das Gewissen und Vorbilder, Ideale, nach denen wir uns richten (das «Über-Ich» nach Freud), und die Vernunft.

Das Leben verhält sich dialektisch. Damit meine ich nicht die philosophische Dialektik von These und Antithese, die eine Synthese sucht, sondern die der Natur innewohnende Gegensätzlichkeit, die nie zu einer Synthese kommt. Wir müssen in der ständigen Spannung dieser Gegensätzlichkeit leben, sie ertragen oder durch Kompromisse erträglich gestalten. (Hegel und Marx versuchten, diese Dialektik in der Geschichte des Menschen nachzuweisen und sahen meines Erachtens fälschlicherweise eine Lösung in einer Synthese, in einem «erlösenden»Endzustand, den es auf dieser Welt nicht geben kann.) Auch unser seelisches Leben ist von dieser Gegenpoligkeit und der dadurch erzeugten Spannung geprägt. Für unsere Betrachtung wichtig sind: Abhängigkeit versus Unabhängigkeit, Gewissen versus Gewissenlosigkeit, Vernunft versus Unvernunft (falsch verstandene Freiheit), Grenzen versus Grenzenlosigkeit, Macht (auch Geldmacht, Karriere und Prominenz) versus Ohnmacht und schliesslich Liebe versus Hass.

Wichtig für meine Betrachtung ist auch der Unterschied zwischen einem reifen und einem unreiferen Gewissen. Jean Piaget hat in seinem Buch «Intelligenz und Affektivität in der Entwicklung des Kindes» (1954) beschrieben, wie die normativen Gefühle sich erst um das siebte Lebensjahr entwickeln. Eine moralische Norm ist auf eine dreifache Weise charakterisiert: «Erstens gilt eine Norm generell für alle analogen Situationen und nicht nur für die gleichen. Zweitens überdauert sie die typischen Situationen und Bedingungen, aus denen sie hervorgeht. Drittens ist sie an das Gefühl von Autonomie gebunden.» Vom zweiten bis zum siebten Lebensjahr «bleibt das Gebot - ähnlich wie unter kognitivem Aspekt an Wahrnehmungsgestalten an bestimmte, konkrete Situationen gebunden, zum Beispiel an die Person, die es gegeben hat. [...] Schliesslich gibt es noch keine «Autonomie〉. Als 〈gut oder 〈böse〉 gilt lediglich, was mit der erhaltenen Anweisung übereinstimmt oder nicht.» Wir reifen von unserer Geburt an jeder in seiner Entwicklungsgeschichte (S. Freud, E. H. Erikson, W. Mertens, J. Piaget, P. Miller et al.) im ständigen Wechselspiel mit seiner Umgebung (vgl. D. W. Winnicott, M. Mahler, D. Stern, J. Lichtenberg et al., sowie die vielen Autoren der systemischen Theorie). Jeder hat seine Geschichtlichkeit (H. G. Gadamer). Freud wurde in seiner Annahme, dass wir in einer Entwicklungsstufe fixiert bleiben können, bis jetzt noch nicht stichhaltig widerlegt, denken wir nur an den Narzissmus (vgl. u.a. A. Miller). Wir können aber als Erwachsene auch in frühere Entwicklungsstufen zurückfallen, regredieren. Wie ich nun beobachten konnte, regrediert damit auch unser Gewissen von einer reiferen in die unreifere Form. So regredieren Menschen unter einer Gewaltherrschaft auf die Stufe der Ohnmacht, wie wir sie alle im ersten Trotzalter (anale Phase nach S. Freud, E. H. Erikson et al.) durcherleben mussten. Neben denen, die aufbegehren oder flüchten, unterwerfen sich die meisten der Macht der «Grossen». Ihr Gewissen regrediert von den normativen Gefühlen zu denen, die Piaget die semi-normativen nennt. Das ist begreif- 
lich, denn die gesetzgebende und hart strafende Autorität ist mit ihren Spitzeln allgegenwärtig. Not- und Katastrophensituationen schweissen Menschen zu voneinander gegenseitig, aber auch von den Helfern abhängigen Personen zusammen, was gut ist, weil dies uns Geborgenheit schafft und der Gemeinschaft überleben und um ihre Verlorenen trauern hilft. In meinen Augen nicht gesund ist es, wenn religiöse und politische Gemeinschaften ein reifes Gewissen und damit auch ein reifes «Ich» im Sinne Freuds gar nicht erst entstehen lassen oder angstmachende und verunsichernde Situationen ausnützen, um die Regression von Menschen in die Abhängigkeit von ihnen zu fördern. Sie berufen sich dabei auf höchste Autoritäten, auf Gott, auf die Bibel, auf Marx und Lenin usw. Sie drohen bei Ungehorsam und abweichenden Meinungen mit Ausschluss, Ächtung und drakonischen Strafen, wie z.B. mit der Strafe Gottes. Diesen ihre Autonomie und ihr freies Entscheiden aufgebenden Menschen steht der faustische Mensch gegenüber, der sich «in den Fängen Mephistos» über alle Skrupel und moralischen Gesetze hinwegsetzt. Der eine gehorcht, der andere fühlt sich absolut frei. Diese beiden wohnen aber auch in jedem von uns. Der Mensch, der in Selbsterkenntnis sein «Ich» auf- und ständig ausbaut, damit meine ich die Fähigkeit, selber zu entscheiden, sich der Realität anzupassen (nicht zu unterwerfen!) und seine Grenzen (oft schmerzlich!) akzeptieren zu lernen, braucht für sein Entscheiden Normen und Ideale, nach denen er sich richtet. Es sind aber seine Normen und seine Ideale. Andere Menschen haben ganz andere, die er sogar schätzen und bewundern lernt, die ihn aber auch ärgern können. Er wird aber nie versuchen, dem anderen seine Normen aufzuzwingen.

So dürfen uns auch staatliche Gesetze, wenn immer möglich, keine ethischen Normen aufzwingen und dadurch unsere Gewissensfreiheit einschränken. Sie müssen aber der Selbstsucht, der Machtgier (nicht dem gesunden Machtstreben), der Unvernunft und der Gewissenlosigkeit - den Fausts und Frankensteins - Grenzen setzen, weil diese der Gemeinschaft schaden. Was heisst das nun in bezug auf unsere Fähigkeit, über Leben und Tod bestimmen zu können? Zu einem Entscheid gehören die Motivation und das Überlegen der Folgen. Der Staat muss die Gesetze über Leben und Tod so abfassen, dass sie dem Menschen seine Gewissensfreiheit belassen, aber dort eingreifen, wo sie für die Gemeinschaft schädlich werden. Eine Grundhaltung, die ausdrückt, du bist frei, du kannst mit Lebendigem - damit meine ich auch Tiere und Pflanzen - umgehen, wie es deinem Gewissen entspricht, kann schädlich sein, weil sie die Achtung und den Respekt vor diesem Lebendigen, aus dem ja auch die Gemeinschaft besteht, nicht konkret unterstützt. Eine Grundhaltung, die uns in unseren Gewissensentscheiden frei lässt, aber zum Ausdruck bringt, dass diese Achtung und dieser Respekt vor dem Lebendigen und dessen gesundes Gedeihen einen wichtigen Wert für die Gesellschaft darstellt, schützt nicht nur, sie fördert die Gemeinschaft. Wie steht es damit bei dem Gesetz über die Fristenregelung? Die Botschaft des jetzigen Gesetzes verstehe ich so: Es ist straffrei und dem Gewissen der Betroffenen anheimgestellt, in Notsituationen bis zur zwölften Schwangerschaftswoche abzutreiben. Die Grundhaltung, die ich von einem solchen Gesetz verlangen würde, hiesse: Einer Frau oder einem Paar ist das Recht gegeben, in Gewissensfreiheit selber zu bestimmen, ob sie eine Schwangerschaft annehmen wollen. Mit konkreten, flankierenden Massnahmen müsste gleichzeitig zum Ausdruck gebracht werden, dass der Gesetzgeber das ungeborene Leben achtet und schützt, indem er konkrete Massnahmen nennt, wie er eine Frau oder ein Paar in einer Notsituation zu unterstützen gedenkt. Das Gesetz müsste Frauen schützen, die unter dem Druck ihres Partners oder der Angehörigen zum Abbruch gezwungen oder massiv gedrängt werden. Unvernünftigen Motivationen müssten einklagbare Grenzen gesetzt werden, z.B. eventuell bleibende Mängel am schönen Körper (in meiner Praxis dreimal erlebt) oder die Gefährdung der Karriere (?, das müsste diskutiert werden). Erwähnenswert wäre auch, dass eine Frau zur Prävention oder selbstverständlich mit entsprechender Unterstützung zum Austragen der Schwangerschaft gezwungen werden kann, wenn sie - sagen wir - zum vierten Mal für einen Abbruch ihren Arzt aufsucht. Frauen oder Paare, die sich für das Austragen entscheiden, müssten belohnt werden. Ähnlich denke ich bei einer Gesetzgebung über die pränatale Diagnostik: Wir müssen den Entscheid, ob sich ein Paar zutraut, ein behindertes Kind aufzuziehen, dem Gewissen dieser Menschen überlassen. Der Gesetzgeber muss aber ausdrücklich kundtun, dass er Behinderte nicht nur lebenswert, sondern für die Gemeinschaft auch als Bereicherung findet, indem er es möglichst vielen Eltern mit konkreten, flankierenden Massnahmen erleichtert, zu einem behinderten Kind ja zu sagen. Er muss diese Menschen, die in dieser wirklich schwierigen Situation zu ihrem Schicksal stehen, ausdrücklich würdigen und belohnen. Bei der Fristenregelung und bei der pränatalen Diagnostik ist für mich dieses Würdigen und Belohnen so 
wichtig, weil ich befürchte, dass Menschen, die in Notsituationen nicht abtreiben, mit den Jahren die Dummen sind oder ihnen sogar Hilfe verweigert werden könnte mit dem Hinweis, hättest du abgetrieben!

Bei vielen von unseren Entscheiden können wir die Folgen nicht absehen und müssen sie nehmen, wie sie sich einstellen. Können wir sie aber erkennen oder ahnen, dürfen wir erst entscheiden, wenn die voraussehbaren Probleme gelöst sind. (Denken wir nur an den Atommüll!) Bei den Retortenbabies wussten wir, dass überzählige Embryonen anfallen. Auch wenn damals die Stammzellenforschung noch nicht so aktuell war wie jetzt, hätte man ahnen können, dass hier für Genforscher ein fast unwiderstehliches Reservoir aufgebaut wird. Heute stellt sich nun die Frage: Sollen wir die kleinen Wesen nächstes Jahr in einer staatlichen Aktion vernichten oder sie den Forschern übergeben? Lieber tot als in den Händen der Forscher, werden viele sagen. Ich aber frage hartnäckig weiter: Wenn die Frauen und Paare verlangen, dass wir bei den Schwangerschaftsabbrüchen ihrem Verantwortungsgefühl vertrauen, warum dürfen wir dies denn unseren Forschern gegenüber nicht ebenso? Sind nicht die meisten von ihnen gewissenhafte, seriöse Menschen? Sicher gibt es Frankensteins, doch so viele sind es nicht, und es ist richtig, wenn man diesen Grenzen setzt. Können wir dies aber als kleiner Nationalstaat? Was nützt es, wenn wir unseren Forschern straffe Zügel anlegen und andere Länder diese viel lockerer lassen oder gar ein grössenwahnsinniger Herrscher Frankensteins um sich schart? Das Problem der Stammzellen- und der Genforschung ist meines Erachtens ein global zu lösendes. Die Forscher der Erde sollen nach ihrem Gewissen selber bestimmen können, wie weit sie gehen wollen. Sie dürfen dies aber nur bis zu einer Grenze, die der Weltgemeinschaft nicht schadet, und zwar in allen Ländern gleich und mit den gleichen Ellen gemessen. Dazu müssen die internationalen Gerichtshöfe ausgebaut und gestärkt werden. Gesetze, auch Weltgesetze, müssten aber nicht nur sanktionieren, was aus Hass, Hybris, Missachtung der Würde und Rücksichtslosigkeit geschieht, sondern auch belohnen, was aus Liebe zur Natur und zu den Menschen, aus dem Akzeptieren von Grenzen, mit Achtung vor der
Würde und mit Rücksicht vollbracht wird. Mit anderen Worten: Sanktionen begegnen Taten aus Motiven, die für die Gemeinschaft schädlich sind, mit Gewalt. Belohnen begegnet Taten aus Motiven, die für die Gemeinschaft förderlich sind, mit Wohlwollen und Unterstützung. Liebe ist schliesslich das Prinzip, das Leben erhält, fördert und im Sinne Jürg Willis (u. a. «Psychologie der Liebe», 2002) koevolutiv wirkt; Hass und mit ihr Gewalt sind die Prinzipien, die Leben zerstören, unterdrücken und regressiv und antievolutiv wirken.

\section{Literatur}

- $\quad$ Erikson EH. Identität und Lebenszyklus. Deutsche Ausgabe. Frankfurt: Suhrkamp; 1966.

- Freud S. Vorlesungen zur Einführung in die Psychoanalyse und Neue Folge. G. W. Band 1 und 15. Frankfurt: Fischer, 1960.

- Gadamer HG. Wahrheit und Methode. Tübingen: Mohr; 1986/93.

- Kolakowski L. Die Hauptströmungen des Marxismus. Band 1. München: Piper; 1977.

- Lichtenberg JD. Psychoanalyse und Säuglingsforschung. Berlin: Springer; 1991.

- Mahler MS. Studien über die drei ersten Lebensjahre. Stuttgart: Klett-Cotta; 1985.

- Mertens W. Entwicklung der Psychosexualität und der Geschlechtsidentität. Band 1. Stuttgart: Kohlhammer; 1994.

- Miller A. Das Drama des begabten Kindes. Frankfurt: Suhrkamp; 1979.

- Miller PH. Theorien der Entwicklungspsychologie. Heidelberg: Spectrum Akademischer Verlag; 1993.

- Piaget J. Intelligenz und Affektivität in der Entwicklung des Kindes. Deutsche Ausgabe. Frankfurt: Suhrkamp; 1995.

- Stern DN. The Interpersonal World of the Infant. New York: Basic Books; 1985.

- $\quad$ von Schlippe A, Schweitzer J. Lehrbuch der systemischen Therapie und Beratung. 5. Auflage. Göttingen: Vandenhoeck \& Ruprecht; 1998.

- Willi J. Ko-Evolution. Reinbek: Rowohlt; 1985.

- Willi J. Psychologie der Liebe. Stuttgart: Klett-Cotta; 2002.

- Willi J. Was hält Paare zusammen? Reinbek: Rowohlt; 1991.

- Winnicott DW. Reifungsprozesse und fördernde Umwelt. München: Kindler; 1974. 